\title{
FORMAÇÃO PARA O SUS NOS CURSOS DE PSICOLOGIA EM ALAGOAS
}

\author{
Simone Maria Hüning \\ Aline Kelly da Silva \\ Wanderson Vilton Nunes da Silva \\ Flávia Maria Santos Lessa
}

\begin{abstract}
RESUMO. Este artigo discute a formação do psicólogo em Alagoas e sua relação com o Sistema Único de Saúde (SUS), a partir da análise dos currículos dos quatro cursos de graduação em Psicologia do Estado. Construímos quatro eixos de análise das disciplinas a partir das ementas, conteúdos programáticos e bibliografias: 1) Clínica, psicopatologia e avaliação psicológica; 2) Biomédicas; 3) Interfaces socioculturais; 4) Fundamentos da pesquisa e atuação em Psicologia. Discutimos as formas de abordagem da saúde, problematizando sua relação com o princípio da integralidade do SUS. Os resultados indicam que na abordagem da saúde predominam conteúdos referentes à identificação e classificação de transtornos e psicopatologias, e uma concepção de sujeito individualista e fragmentada, baseada no modelo biomédico. Por sua vez, a abordagem da integralidade, quando presente nos currículos, possui o potencial de nortear mudanças na graduação, vinculando-se a cenários diversificados, como atuação multiprofissional e capacitação profissional generalista.
\end{abstract}

Palavras-chave: Formação; psicologia; Sistema Único de Saúde.

\section{PROFESSIONAL EDUCATION FOR NHS IN PSYCHOLOGY COURSES IN ALAGOAS}

ABSTRACT. This article discusses the academic education of psychologists in the province of Alagoas and its relationship with Brazilian National Health System (SUS), from the analyzes of curricula of the four undergraduate Psychology courses of the province. We built four lines of analysis of subjects from the summaries, syllabuses and bibliographies: Clinical, psychopathology and psychological evaluation; Biomedical; Socio and cultural interfaces; Fundamentals of research and work in Psychology. We discussed ways to approach health, problematizing their relationship with the integrality principle in SUS. The results indicate that in the approach to health there is the predominance of content focused on the identification and classification of disorders and psychopathology, and an individualistic and fragmented conception of the subject based on the biomedical model. In turn, the approach of integrality, when present in curricula, has the potential to guide changes in undergraduate courses, binding to diverse scenarios, multidisciplinary and a generalist professional education.

Key words: Academic education; psychology; Brazilian National Health System.

\section{FORMACIÓN PARA EL SUS EN LOS CURSOS DE PSICOLOGÍA EN ALAGOAS}

RESUMEN. En este artículo se discute la formación de los psicólogos en Alagoas y su relación con el Sistema Único de Salud (SUS), basado en el análisis de los planes de estudio de los cuatro títulos de grado en psicología por parte del Estado. Hemos construido cuatro líneas de análisis de temas, desde los menús, planes de estudios y bibliografías: evaluación de la psicopatología clínica y psicológica, Interfaces biomédicos, socioculturales, fundamentos de la investigación y el trabajo en Psicología. Discutimos maneras de abordar la salud y reflexionando su relación con lo principio de integridad del SUS. Los resultados indican que en el enfoque de la salud hay predominancia de los contenidos dirigidos en la identificación y clasificación de los trastornos y la psicopatología, y una concepción de sujeto individualista y fragmentada en el modelo biomédico. A su vez, el enfoque de la integralidad, cuando viene presente en el plan de estudios, tiene el potencial para orientar los cambios en el grado, la unión a diversos escenarios y multidisciplinarios y a la formación profesional generalista.

Palabras-clave: Formación; psicología; Sistema Único de Salud.

Doutora em Psicologia pela PUCRS, professora adjunta da Universidade Federal de Alagoas (UFAL).

\# Mestranda em Psicologia pela UFAL.

I Mestre em Psicologia pela UFAL.

æ Psicóloga formada pela UFAL. 
Este trabalho tem como objetivo discutir a formação do psicólogo em Alagoas destinada à inserção no Sistema Único de Saúde (SUS), através da análise dos currículos dos quatro cursos de Psicologia do estado.

A inserção da Psicologia no campo da saúde ocorre, sobretudo, a partir da década de 1980, em um contexto marcado pela crise econômica, que afastou boa parte da população de consultórios particulares, e pelos questionamentos que a própria Psicologia brasileira começou a fazer sobre si mesma em relação a seus modelos teóricos e à atuação profissional diante da realidade do país (Dimenstein, 1998). Não obstante, de acordo com Lima (2005), embora situados nos serviços públicos de saúde, os profissionais da área reproduziam nas Unidades Básicas de Saúde o modelo clínico tradicional, limitado à compreensão da dimensão intraindividual dos fenômenos.

Além disso, a formação na área de Psicologia pauta-se, historicamente, por modelos de atuação individualizantes e elitistas, e por dicotomias - como, por exemplo, a cisão indivíduo $\mathrm{x}$ sociedade, clínica $\mathrm{x}$ política, interno $\mathrm{x}$ externo, etc. - sustentadas pela pretensão de atingir uma suposta neutralidade científica similar à das Ciências Naturais (Dimenstein, 2000; Benevides, 2005; Foucault, 2002). Como afirmam Nascimento, Manzini e Bocco (2006), os cursos de graduação ainda têm preparado profissionais, tradicionalmente, para a atuação em consultórios, a partir de uma concepção de clínica anistórica e acrítica, embora possam também ser encontradas perspectivas clínicas que buscam romper com tais concepções.

A necessidade de mudanças na formação em saúde vem sendo amplamente debatida no país, especialmente com a aprovação das Diretrizes Curriculares Nacionais, em 2004, a partir das quais se compreende que a formação dos profissionais de saúde deve atender às necessidades sociais, com ênfase no SUS (Ceccim \& Feuerwerker, 2004).

Desse modo, a fim de investigar a formação desses profissionais em Alagoas, realizamos, entre os anos de 2009 e 2011, uma pesquisa ${ }^{1}$

\footnotetext{
A pesquisa "Formação do Profissional da Psicologia em Alagoas e o SUS" foi realizada com financiamento do Edital PPSUS 2008-2009 (MS/CNPQ/FAPEAL/SESAU$A L)$ e o apoio do CNPq, da FAPEAL e da UFAL, através de bolsas de Iniciação Científica. Consentiram formalmente com a participação na pesquisa os quatro cursos de Psicologia existentes no Estado, dos quais dois são de instituições públicas federais e dois de instituições privadas.
}

que contemplou a análise de ementas, conteúdos programáticos e bibliografias dos currículos dos quatro cursos de graduação, com vista a identificar como eles se articulam ao princípio da integralidade, por ser este um dos princípios norteadores da proposta de atenção em saúde vinculada ao SUS, e a analisar quais são os modos e os cenários de intervenção em destaque nesses currículos. Trabalhamos somente com as disciplinas obrigatórias dos cursos, porque em alguns cursos não havia regularidade na oferta das disciplinas eletivas, e muitas delas, embora constassem nas grades curriculares, nunca haviam sido oferecidas e possuíam apenas a ementa.

\section{MEDOTOLOGIA: CURRÍCULOS E EIXOS TEMÁTICOS DE ANÁLISE}

A análise que empreendemos neste trabalho resulta dos dados produzidos a partir das matrizes curriculares e do detalhamento das disciplinas dos quatro cursos de Psicologia do estado. Nossa compreensão, em consonância com Veiga-Neto (2002), é de que os currículos constituem-se como artefatos educacionais que retiram elementos de uma cultura e os escolarizam. Neste sentido, o currículo possui estreitas relações com os processos políticos, econômicos e sociais de uma determinada sociedade.

Em nosso entendimento, esse currículo se relaciona com a cultura e com questões sociais, uma vez que, ao procurar atender às necessidades do público para 0 qual é destinado, elege certos conteúdos, excluindo outros. Particularmente na história da Psicologia, é possível visualizar a presença de marcas históricas e políticas do contexto brasileiro na constituição e modificação dos currículos, desde o ano de regulamentação da profissão (1962) até hoje (Scarparo \& Guareschi, 2007).

A partir desse entendimento, propusemo-nos a estudar os currículos dos cursos de graduação em Psicologia de Alagoas, atentando para as transformações no campo político e cultural, especialmente aquelas impulsionadas pela criação e busca da consolidação do SUS, cuja promulgação, em 1990, rompe com a lógica assistencialista e centrada na cura de doenças, vigente até então. Com a implantação desse Sistema, a saúde passa a ser considerada como direito de todos e dever do Estado, ao qual cabe assegurar a organização de uma rede de serviços 
hierarquizada e descentralizada, a partir de um modelo de saúde baseado em ações de tratamento, promoção e prevenção. Além disso, as práticas em saúde passam a requerer articulação entre saberes de diferentes campos do conhecimento, inclusive aqueles advindos das Ciências Humanas e Sociais, para a efetivação dos princípios do SUS. Busca-se, assim, romper com o modelo médico hegemônico, centrado em práticas biologicistas e hospitalocêntricas, e amplia-se o conceito de saúde, que passa a ser entendida como resultado de condições de vida, e não mais como simples ausência de adoecimento.

Tomamos os currículos como objeto de estudo por considerarmos que eles são os documentos que referendam as práticas de formação e nos permitem ter contato com aquilo que os compõe em termos de objetivos e conteúdos, possibilitando uma visão panorâmica do seu conjunto de disciplinas. Além disso, é nesse material que se encontra o que se pode chamar de "oficial" em relação a seus conteúdos e objetivos.

A partir da obtenção de ementas, conteúdos programáticos e bibliografias de cada disciplina dos cursos, os dados foram organizados em quadros que possibilitaram melhor visualização dos materiais para análise. Em seguida estas foram agrupadas em eixos temáticos de análise, construídos a posteriori. O procedimento inicial que permitiu a inclusão de cada disciplina em um eixo específico foi a identificação do disposto na ementa, no conteúdo e na bibliografia indicada, atentando-se para a abordagem predominante em cada disciplina. Tomamos como referência, para a construção e a análise dos eixos, o estudo realizado por Guareschi, Dhein, Reis, Machry e Bennemann (2010) acerca dos currículos de Psicologia no Rio Grande do Sul, o qual problematiza a formação para o SUS. Desse modo formulamos quatro eixos, estruturados pelos principais focos das disciplinas curriculares analisadas: 1) Clínica, psicopatologia e avaliação psicológica; 2) Biomédicas; 3) Interfaces socioculturais; 4) Fundamentos da pesquisa e atuação em Psicologia. $O$ procedimento seguinte buscou apontar as relações entre os programas das disciplinas obrigatórias e as concepções de saúde e de integralidade estabelecidas em documentos e produções acadêmicas que discutem a Psicologia, a formação e o SUS. Nessa etapa a análise não só voltou-se para o número de disciplinas em cada Eixo, mas buscou problematizar o modo como os conceitos são trabalhados e suas relações com a formação de psicólogos para o trabalho no SUS.
A seguir apresentamos a descrição de cada eixo.

\section{Clínica, psicopatologia e avaliação psicológica}

Este eixo foi composto por disciplinas organizadas em torno de abordagens da Psicologia que operam com a concepção de subjetividade como relativa a uma instância de interioridade psicológica, ou que, mesmo sem operar com a definição de subjetividade, focalizam conceitos como sujeito psíquico, processos psicológicos e psiquismo. Envolve disciplinas que instrumentalizam os estudantes para o conhecimento das instâncias internas dos sujeitos e as práticas de intervenção que a elas correspondem. São aqui contempladas disciplinas que constroem as bases para a atuação clínica, a identificação de processos de desenvolvimento do sujeito, o conhecimento e utilização de ferramentas de avaliação psicológica e a análise de processos psicológicos básicos e psicopatologias. Pode-se dizer, de forma resumida, que este eixo constitui-se de disciplinas estruturadas em torno do que Prado Filho e Martins (2007) definem como o "reino da subjetividade", ou aquilo que envolve "o psiquismo, a cognição, a 'mente', a consciência, a identidade, o self, mas também as percepções, as interpretações, e uma certa dimensão 'intrapsíquica', das emoções, do desejo, do inconsciente" (p. 14). O trabalho de Spink e Matta (2007) também contribui para a delimitação desse eixo, ao apontar para perspectivas da Psicologia baseadas na "problemática do sujeito", traduzida em modelos frequentemente caracterizados por um viés clínico de avaliação, aferição dos processos psicológicos básicos e ajuste ao meio social em que as pessoas estabelecem suas atividades cotidianas, com a utilização de testes de avaliação psicológica e outros modos de aferição.

\section{Biomédicas}

Este eixo identifica as disciplinas relacionadas às ciências médicas e biológicas que dialogam com a Psicologia na constituição de seus saberes e práticas profissionais. Articulam-se aqui as disciplinas cujas ementas e conteúdos constituem-se com base nos campos da Biologia e Medicina que compõem os currículos de formação em Psicologia.

\section{Interfaces socioculturais}

Neste eixo propomos uma leitura do currículo que visualize as disciplinas que promovem interfaces com questões 
socioculturais. O eixo contempla disciplinas da Psicologia que apresentem abordagens interdisciplinares e proponham a análise de questões vinculadas à sociedade e à cultura, como a dimensão de instituições e fenômenos sociais contemporâneos. Para compor este eixo, não elencamos as disciplinas que tomam como foco a interioridade psicológica, mas aquelas que dão ênfase às dimensões política, social, cultural e econômica, entre outras que atravessam a produção de subjetividade na sociedade contemporânea e que, por isso mesmo, vinculam-se à construção de modos alternativos de compreensão da subjetividade. Integram ainda o eixo outras disciplinas das Ciências Humanas que, mesmo não se caracterizando como psicológicas, contribuem para a formação do psicólogo.

\section{Fundamentos da pesquisa e atuação em Psicologia}

Este eixo é composto por disciplinas voltadas aos fundamentos da pesquisa e atuação profissional do psicólogo. Estas disciplinas abordam a história da Psicologia e suas escolas, englobando as dimensões epistemológica, teórica e metodológica. Contemplam ainda as orientações e regulamentações nos processos de produção do conhecimento e do exercício da Psicologia.

Os procedimentos de análise dos elementos curriculares (ementas, conteúdos e bibliografias) nos permitiram visualizar que conteúdos e abordagens relativos à saúde vêm sendo priorizados nos cursos. A distribuição das disciplinas em cada um dos eixos e a prevalência de alguns eixos em relação a outros em cada curso indicaram maior ou menor aproximação desses currículos, com uma formação consoante com a proposta do SUS, baseada na abordagem integral do processo saúde-doença e no trabalho interdisciplinar e intersetorial.

\section{RESULTADOS: ARTICULAÇÕES DA PSICOLOGIA E SAÚDE NOS CURRÍCULOS EM ALAGOAS}

Após o agrupamento das disciplinas nos eixos temáticos, focamos a identificação, em cada Eixo, das disciplinas que faziam referência às ementas, conteúdos ou bibliografias referentes à saúde, sem uma concepção de saúde pré-definida, considerando apenas a menção a esta ou aos processos de saúde/adoecimento, cuidado, tratamento e/ou prevenção. Aspectos socioculturais, entre outros que, na proposta do SUS, são constituintes da saúde, foram considerados na medida em que se estabelecesse nos programas examinados alguma relação desses com a saúde.

A partir disso, obtivemos o gráfico abaixo.

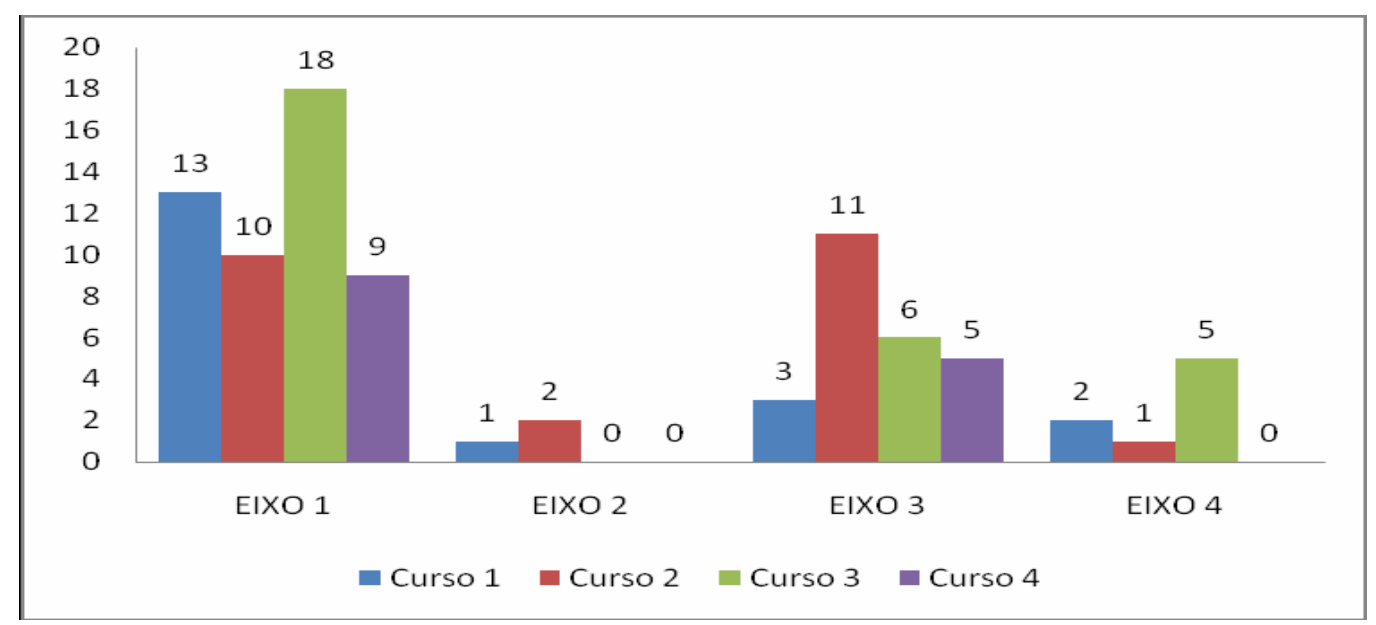

Gráfico 1. Disciplinas que remetem à saúde, nos quatro cursos, por Eixo. Os eixos se referem a: Eixo 1 Clínica, psicopatologia e avaliação psicológica; Eixo 2 - Biomédicas; Eixo 3 - Interfaces socioculturais; e Eixo 4 - Fundamentos históricos e epistemológicos.

Nesse gráfico pode-se observar que, na maioria dos cursos, o tema da saúde é trabalhado principalmente no eixo 1, com exceção do Curso 2, no qual a saúde aparece distribuída nas disciplinas dos eixos 1 e 3 . Isso nos leva a pensar que a formação nesse curso possui um caráter voltado tanto para a questão da patologia como para as questões socioculturais. 
No eixo 1, ao analisarmos as ementas das disciplinas que o compõem, encontramos conteúdos voltados às questões do diagnóstico, da patologia e da avaliação, a partir de perspectivas tradicionais, afastando-se da proposta do SUS de se levarem em conta os determinantes sociais do processo saúdedoença, isto é, de considerar os fatores socioculturais e os processos de subjetivação que perpassam a produção de saúde e de doença. Os conteúdos desse eixo privilegiam um olhar limitado à cura de doenças, não levando em conta a questão da promoção da saúde.

Merece destaque o baixo número de disciplinas no eixo 2 em todos os cursos, o que chama a atenção pelo grande número de trabalhos que discutem a formação em Psicologia e a influência do modelo biomédico nesta área. Não obstante, ao utilizarmos uma análise qualitativa destes dados em que consideramos, além do número de disciplinas por Eixo, os conceitos e referenciais teóricos trabalhados em cada curso, balizados tanto por tais descrições como pelas bibliografias indicadas nas disciplinas - notamos que, apesar do baixo número de disciplinas neste eixo, ele aparece incorporado aos outros, como na disciplina Psicopatologia Geral do Curso 2, que aborda a questão dos sintomas psicofisiológicos. Essa disciplina, apesar de estar localizada no eixo 1, utiliza-se de bases biológicas para validar seu conteúdo. Ela foi posta nesse eixo por nela prevalecerem conteúdos referentes à clínica, à psicopatologia e à avaliação psicológica, em que as questões biomédicas aparecem como um suporte para a relação mente-corpo.

No eixo 3 foram observadas abordagens diretas ao SUS e ao conceito de saúde que ele instaura. Questões como a Reforma Psiquiátrica, a atenção integral à saúde, a prevenção e a promoção da saúde aparecem dispersas em disciplinas que compõem esse eixo. É nele que se situa o núcleo de discussão que converge para o que propõe o SUS, tanto em termos de concepções como de modos de atuação em saúde, destacando-se o trabalho em equipes interdisciplinares e a necessidade de articulações intersetoriais na rede de serviços. Já no eixo 4, os conteúdos que sobressaem estão ligados à constituição da Psicologia enquanto ciência e às diferentes bases epistemológicas desse campo de saber, bem como à instrumentalização metodológica necessária à produção de conhecimento e à prática profissional.

Essa distribuição das disciplinas nos permite visualizar que os cursos de graduação em Psicologia no Estado de Alagoas, a exemplo do que ocorre historicamente em todo o país, têm privilegiado a abordagem da clínica, da psicopatologia e da avaliação psicológica, e é a partir principalmente desse enfoque que a saúde tem sido trabalhada. Apesar dessa dominância, percebemos algumas transformações, evidenciadas principalmente no currículo do Curso 2, com a inclusão equitativa de questões relativas às interfaces socioculturais na abordagem da saúde.

Posteriormente à elaboração dos eixos, selecionamos as disciplinas que remetem à saúde, considerando como tais aquelas que tratassem dos modos de cuidado e da promoção da vida. A seguir, discutimos as concepções de saúde de forma integrada em relação aos quatro cursos estudados, para evitar a produção individual de "avaliações" a respeito dos cursos.

\section{CONCEPÇÕES DE SAÚDE}

Com a criação do Sistema Único de Saúde em 1990 houve uma transformação no conceito de saúde. Baseando-se nos princípios e diretrizes do SUS, os serviços de saúde deixam de ser operados numa lógica puramente assistencialista, havendo ampliação destes para a prevenção, proteção e promoção à saúde. Diante disto, a saúde, "entendida até então pela ausência de doenças ou sintomas, passa a compreender questões de saúde, como direito do cidadão na promoção das condições de vida, relacionando-se aos modos de ser e estar no mundo, ou seja, saúde como um dispositivo de modos de subjetivação" (Guareschi, Dhein, Reis, Machry \& Bennemann, 2009, p. 37). A saúde passa de um conceito que remetia puramente ao corpo e começa a englobar outros aspectos, como a subjetividade, o social, o cultural, o histórico e o econômico, embora muitas vezes estes ainda sejam vistos de forma fragmentada e dicotomizada.

Observamos que o conceito de saúde não esteve sempre presente no campo da Psicologia e não há como dizer com exatidão o momento em que a Psicologia passou a construir saberes acerca da saúde; contudo, podem-se desenvolver reflexões acerca das condições e possibilidades que levaram a Psicologia a voltar o olhar para a saúde e dos contextos em que estas se deram. Para Spink (2003), a Psicologia ingressa na classificação das profissões reconhecidas como da área de saúde por meio 
da aplicação de conhecimentos técnicos derivados da experiência clínica tradicional, a qual se centra em um indivíduo abstrato e anistórico, sem uma reflexão sobre esta transposição de técnicas de um campo para outro, mas com base, principalmente, no modelo médico hegemônico de identificação de doenças que podem ser vistas e diagnosticadas pelos sintomas. No caso da Psicologia, os sintomas referiam-se aos transtornos e desvios mentais.

Para Guareschi e cols. (2009), a inserção da Psicologia na saúde se deu através do desenvolvimento e utilização de instrumentos, práticas e saberes que, aliados à Medicina Social, proporcionaram o estudo epidemiológico de aferição de patologias e doenças descritivas e na classificação de grupos de risco e de populações vulneráveis, caracterizando uma perspectiva de saúde baseada no discurso higienista. Assim, a Psicologia, por meio dos testes e da avaliação psicológica, ingressou no campo da saúde pautada por um modelo biologicista, o qual buscava identificar distúrbios e transtornos para a compreensão e tratamento de doenças.

A análise dos currículos dos cursos do Estado, de modo consonante com a trajetória histórica da Psicologia no campo da saúde, evidenciou que as disciplinas inseridas no eixo 1 (Clínica, psicopatologia e avaliação psicológica) trazem em seus conteúdos temas que abordam a psicopatologia e o psicodiagnóstico com enfoque predominante na identificação e classificação de doenças, muitas vezes embasado no saber psiquiátrico. Tais disciplinas buscam definir o que é da ordem do normal e do patológico, a partir da personalidade e do desenvolvimento psíquico, motor e orgânico. A concepção de saúde nestas disciplinas remete à fragmentação, dicotomizando o sujeito em mente e corpo. O processo saúde e doença é pouco discutido, aparecendo de forma tímida, articulado à clínica ampliada. Acredita-se que a abordagem da clínica ampliada, mesmo que ainda periférica, possibilita a construção de uma perspectiva de saúde mais ampla. Em alguns casos, neste eixo as disciplinas que se propunham à avaliação psicológica também traziam em seus conteúdos referências à discussão das questões éticas que devem se implicar neste processo e as limitações dos instrumentos utilizados para tal; no entanto, mais uma vez essa discussão apresentou-se de forma bastante pontual.

O eixo 2, composto pelas disciplinas de caráter biomédico, embora seja composto por poucas disciplinas, também enfatiza a identificação e classificação de psicopatologias reproduzindo a dicotomia mente-corpo e apoiando-se no saber médico-psiquiátrico ao se propor a tratar das desordens por meio de medicamentos e das relações entre a biologia, a bioquímica, a psicofarmacologia e a psicopatologia. A concepção de saúde que está sendo trabalhada nesta disciplina, assim como no eixo 1 , privilegia a fragmentação do sujeito. $O$ sujeito não é visto de forma integral, ele é dicotomizado em orgânico e psíquico. Neste eixo não é feita nenhuma menção a fatores sociais e culturais que poderiam relacionar-se tanto à produção das psicopatologias quanto aos modos farmacológicos de intervenção, os quais constituem o conteúdo principal. Tais aspectos nos permitem pontuar que, nesses casos, o saber médico é utilizado sem uma reflexão crítica das implicações de sua transposição para o campo da Psicologia, destacando-se que não se trata aqui de uma prática de diálogo interdisciplinar, mas de uma sobreposição de saberes. Ressaltamos que, mesmo sendo este eixo composto por poucas disciplinas, deve-se atentar para o modo com esse modelo biomédico se dispersa e constitui as práticas psicológicas e concepções de saúde que operam no campo psicológico, o que pode ser especialmente constatado a partir da análise das disciplinas do eixo 1.

No eixo 3 (Interfaces socioculturais) percebe-se a presença de um conceito ampliado de saúde. Em contraposição ao que prevalece nos dois eixos anteriores, encontramos aqui temas referentes aos diferentes modelos e aos diferentes contextos de atuação em saúde. Este aspecto é relevante por considerar práticas da Psicologia no campo da saúde que vão além dos consultórios e dos hospitais. Algumas disciplinas trazem em seus conteúdos a atuação da Psicologia voltada para a saúde em comunidades, escolas, na área organizacional, na rede de Atenção Primária, dentre outras. É nesse eixo que se situam discussões vinculadas especificamente ao conceito de saúde, bem como a abordagem da saúde e da doença como processos relacionados a fatores sociais, econômicos e culturais. Diante disto, é possível construir uma perspectiva de sujeito integral e não dicotomizado. Isto implica em práticas de saúde que abarquem esse sujeito integral por meio de uma atuação baseada na integralidade e em equipes interdisciplinares, para que o sujeito seja visto em sua totalidade. Os conteúdos que abordam as equipes inter e multidisciplinares e os diferentes 
contextos de atuação auxiliam na construção desta prática.

Em relação ao eixo 4 (Fundamentos da pesquisa e atuação em Psicologia), mais uma vez encontramos referências à identificação e diagnóstico de transtornos, porém com inserção de reflexões sobre as questões éticas implicadas no diagnóstico. A pesquisa aparece relacionada ao campo da saúde em uma disciplina desse eixo. No curso em que há mais disciplinas relacionadas à saúde no eixo 4, destacam-se temas referentes à atuação em equipes multi, inter e transdisciplinares, à atuação em saúde em diferentes contextos, à promoção da saúde e à integralidade.

Em síntese, no que concerne às concepções de saúde trabalhadas nos currículos dos quatro cursos de graduação em Psicologia do Estado, percebe-se a predominância de conteúdos relacionados à identificação e classificação de transtornos e psicopatologias em um viés individualizante, a partir da concepção de sujeito fragmentado e, muitas vezes, numa perspectiva baseada nos saberes médicos psiquiátricos. Estes conteúdos podem contribuir para uma concepção de saúde fragmentada, focalizando os aspectos psicológicos e biológicos em detrimento dos demais que se relacionam ao processo de saúde-doença. Pode-se observar também que, embora algumas disciplinas abordem fatores como o social, o cultural, o econômico, estes ainda aparecem de forma isolada e, portanto, reproduzindo as fragmentações.

\section{A INTEGRALIDADE COMO PRINCÍPIO NORTEADOR DE MUDANÇAS}

A integralidade é tida, pelo SUS como um princípio fundamental, que garante ao usuário ações de promoção, prevenção, tratamento e acesso a todos os níveis de complexidade do Sistema Único de Saúde (Brasil, 2009). Brito-Silva, Bezerra e Tanaka (2012) afirmam que a integralidade implica 0 reconhecimento da complexidade e das necessidades de cada indivíduo e ressaltam a relevância desse princípio para a operacionalização do SUS. Para Mattos (2001), esse conceito pode ser também entendido como um conjunto de valores pelos quais se luta e/ou uma imagem-objetivo que, ao mesmo tempo, evidencia aquilo que criticamos no presente $e$ indica a direção que almejamos no que se refere à transformação da realidade. Para esse autor, a integralidade, enquanto imagem-objetivo, é polissêmica, assume vários sentidos. Ceccim e Feuerwerker (2004) argumentam que a integralidade deve ser um eixo norteador de mudanças na graduação das profissões de saúde, por promover ações de atenção à proposta política do SUS, sustentando-se através da articulação com as redes.

As redes de serviço e atenção em saúde surgem para dar suporte ao atendimento integral, em que o usuário, ao procurar atendimento, não o receba de forma isolada, fragmentada, mas de forma continuada e integral. Para a efetivação de serviços em rede, vale ressaltar a importância do diálogo e da troca de informações entre os setores de saúde como ponto desencadeador do trabalho em rede nos serviços em saúde (Brasil, 2009).

Com o movimento em busca da consolidação do SUS, os trabalhadores da saúde, incluindo os psicólogos, começam a enfrentar desafios, em função, entre outros motivos, da predominância do modelo hegemônico ainda imperante no campo da saúde e da própria Psicologia (Spink \& Matta, 2007). Em contrapartida, a integralidade surge como uma crítica a uma atitude fragmentária, baseada nas especializações que reduzem 0 sofrimento das pessoas apenas a aspectos biológicos e restringem as práticas dos profissionais de saúde à atenção individual e curativa (Araújo, Miranda \& Brasil, 2007).

A análise da abordagem da integralidade nos currículos dos cursos de graduação em Psicologia do Estado de Alagoas partiu do quadro inicial de disciplinas que faziam referência à saúde, já apresentado no gráfico 1 deste trabalho. Entre essas disciplinas destacamos aquelas que se relacionavam à integralidade. As informações apresentadas neste estudo foram inicialmente organizadas a partir das palavras-chave que apareciam nos currículos, tais como CRAS, CAPS, biopsicossocial, diagnóstico, promoção e prevenção, entre outras. Delimitamos, então, três dimensões associadas à abordagem da integralidade no SUS: 1) Articulação de políticas públicas/intersetorialidade; 2) Complexidade do processo saúde-doença do sujeito; 3) Integração de ações e serviços de promoção, prevenção e tratamento. Como critério de inclusão das disciplinas relacionadas à saúde na perspectiva da integralidade, traçou-se uma matriz a partir do que o SUS define como integralidade. Assim, para que a disciplina fosse incluída nessa etapa, ela deveria contemplar pelo menos uma das dimensões mencionadas acima.

$\mathrm{Na}$ primeira dimensão apontada (Articulação de políticas públicas/intersetorialidade) buscou-se identificar nas ementas e conteúdos programáticos como as instituições de Ensino Superior articulam 
as políticas públicas de saúde com os demais setores, cientes de que "a intersetorialidade como prática de gestão na saúde [...] e diferentes setores do governo atuam na produção da saúde na formulação, implementação e acompanhamento de políticas públicas que possam ter impacto positivo sobre a saúde da população" (Brasil 2009, p.193).

No que diz respeito à dimensão Complexidade do processo saúde-doença do sujeito, buscamos identificar temas relacionados à etiologia de tal processo e/ou à intervenção na coletividade, no indivíduo ou em qualquer elemento do contexto que compõe o complexo de determinantes e condicionantes dos processos de saúde e doença (Vasconcelos \& Pasche, 2009).
Por último, quanto à dimensão Integração de ações e serviços de promoção, prevenção $e$ tratamento, buscou-se mapear nos currículos questões voltadas ao atendimento que deve ser feito para à saúde, e não apenas à doença, a partir do pressuposto de que o atendimento deve ser feito também para erradicar causas e diminuir riscos, além de tratar os danos.

Para a organização dos dados, criamos um quadro que relaciona os eixos elaborados na pesquisa com as três dimensões construídas em torno do princípio de integralidade do SUS. Nesse quadro apresentamos os termos que se relacionam a cada uma dessas intersecções.

\section{Dimensões da integralidade nos quatro Eixos de análise}

\begin{tabular}{|c|c|c|c|}
\hline & $\begin{array}{c}\text { Articulação de Políticas Públicas } \\
\text { (Intersetorialidade) }\end{array}$ & $\begin{array}{l}\text { Complexidade do Processo Saúde- } \\
\text { Doença do Sujeito }\end{array}$ & $\begin{array}{l}\text { Integração de Ações e Serviços } \\
\text { de Promoção, Prevenção e } \\
\text { Tratamento }\end{array}$ \\
\hline $\begin{array}{|lrr|}\text { Eixo } & 1 & \text { (Clínica, } \\
\text { Psicopatologia } & \text { e } \\
\text { Avaliação } & \text { Psicológica) }\end{array}$ & & $\begin{array}{l}\text { - Desenvolvimento físico, psicomotor, } \\
\text { cognitivo, moral; } \\
\text { - Fases do desenvolvimento } \\
\text { Psicossocial; } \\
\text { - Aspectos biológicos do } \\
\text { desenvolvimento; } \\
\text { - Epidemiologia Social; } \\
\text { - Intervenção Psicomotora em } \\
\text { contextos educativo, cultural e } \\
\text { esportivo; } \\
\text { - Diagnóstico } \\
\text { - Multidisciplinar }\end{array}$ & \\
\hline Eixo 2 (Biomédicas) & & $\begin{array}{l}\text { - Neurociência; } \\
\text { - Anatomia do corpo humano; } \\
\text { - Bioquímica; } \\
\text { - Doenças Mentais; } \\
\text { - Avaliação Neurológica. }\end{array}$ & \\
\hline 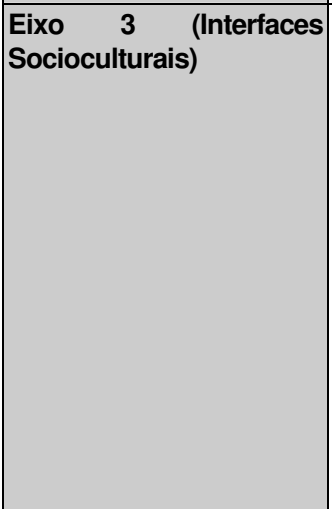 & $\begin{array}{l}\text { - Intersetorialidade e Rede Social; } \\
\text { - Rede Básica; } \\
\text { - Saúde Pública; } \\
\text { - Serviços de Saúde Mental e } \\
\text { - Coletiva; } \\
\text { - Psicologia na diversidade dos } \\
\text { processos educativos; } \\
\text { - Níveis de atenção e saúde; } \\
\text { - Saúde Coletiva; } \\
\text { - Políticas Públicas em Saúde; } \\
\text { - Políticas Educacionais e de } \\
\text { assistência social; } \\
\text { - SUS. }\end{array}$ & $\begin{array}{l}\text { - Fatores Sócio- Econômicos e } \\
\text { culturais; } \\
\text { - Saúde, educação e segurança; } \\
\text { - Equipes multidisciplinares; } \\
\text { - Os diferentes contextos socioculturais; } \\
\text { - Cenários de práticas em Alagoas; } \\
\text { - Interdisciplinaridade; } \\
\text { - Atenção em comunidade; } \\
\text { - Noções culturais e sociais; } \\
\text { - Transdisciplinaridade; } \\
\text { - Saúde Psicossocial. }\end{array}$ & $\begin{array}{l}\text { - Prevenção e Promoção de } \\
\text { Saúde; } \\
\text { - Modelos de atenção e } \\
\text { possibilidades de ação social; } \\
\text { - Serviços de saúde e } \\
\text { necessidades sociais; } \\
\text { - Qualidade de vida; } \\
\text { - Atenção Integral na saúde e } \\
\text { social; } \\
\text { - Psico-higiene. }\end{array}$ \\
\hline $\begin{array}{l}\text { Eixo } 4 \text { (Fundamentos da } \\
\text { Pesquisa e Atuação em } \\
\text { Psicologia) }\end{array}$ & $\begin{array}{l}\text { - Saúde Pública e Atenção Básica; } \\
\text { - CAPS,CAPS-AD, CAPSI, } \\
\text { Residência Terapêutica; } \\
\text { - Política Nacional de Assistência } \\
\text { Social (SUAS, CRAS) e o SUS. }\end{array}$ & - Equipes multi/inter/transdisciplinares & $\begin{array}{l}\text { - Promoção da saúde; } \\
\text { - Atuação psi em programas de } \\
\text { Promoção e qualidade de vida. }\end{array}$ \\
\hline
\end{tabular}

Quadro 1. Relaciona os Eixos da pesquisa com as dimensões da integralidade, estabelecidas a partir do que determina o SUS. No quadro, são apresentados os termos-chave que aparecem nos currículos relacionados à integralidade. 
Nas discussões que seguem analisamos 0 quadro acima, problematizando os currículos na interface com o SUS em cada uma das dimensões apontadas.

\section{Articulação de políticas públicas/intersetorialidade}

Essa dimensão caracteriza-se por compreender discussões em torno das redes de assistência e de saúde em conjunto com as políticas públicas e a articulação entre setores para garantir a continuidade das redes. A escolha das disciplinas que integrariam esta dimensão foi realizada a partir de termos que remetiam a dimensões da integralidade direcionadas às redes de assistência nas políticas públicas.

Ao se tentar compreender a dimensão 1, torna-se importante a discussão acerca da intersetorialidade no trabalho interdisciplinar das ações de saúde, priorizando os diálogos nos ambientes de trabalho a partir da construção de equipes, com a ampliação dos discursos e possibilidades de intervenção psicossocial. No âmbito da formação em Psicologia, isso se relaciona às articulações entre ensino-serviço e dimensões da integralidade na formação, o que traz à tona a importância, na formação, do diálogo entre os saberes e entre os usuários dos serviços de saúde.

A dimensão 1, presente nos eixos 3 e 4, caracteriza-se por apresentar disciplinas que remetem à atuação do psicólogo nos serviços públicos de saúde.

\section{Complexidade do processo saúde-doença do sujeito}

Todos os eixos são contemplados nessa dimensão, aparecendo palavras-chave consonantes a partir de concepções biológicas relacionadas ao diagnóstico. Notamos que esta é a única dimensão contemplada nos eixos 1 e 2. Tais eixos dialogam entre si, visto que a psicopatologia se constitui a partir das ciências biológicas, pois para a compreensão do diagnóstico de transtornos mentais é necessária uma avaliação dos processos bioquímicos. As questões abordadas nestes eixos circulam em torno da descrição biológica do processo saúdedoença do sujeito. No que se refere aos eixos 3 e 4, essa dimensão se relaciona à caracterização de cenários de intervenção e à atuação em equipes interdisciplinares.
Integração de ações e serviços de promoção, prevenção e tratamento

Por fim, procurou-se analisar a abordagem da integralidade a partir da dimensão que articula ações e serviços de promoção, prevenção e tratamento. Segundo a Cartilha do SUS de A a Z (Brasil, 2009), o princípio da integralidade é efetuado na prática através da garantia ao usuário de uma atenção que abranja as ações de promoção, prevenção, tratamento e reabilitação. Tais ações, quando articuladas com outros setores, contribuem para a melhoria de condições de vida e saúde da população, sendo sustentadas por parcerias e ações interdisciplinares.

Os termos encontrados no eixo 4 foram fundamentais para se perceber a mudança que vem acontecendo no cenário alagoano, na perspectiva da integralidade, por ser um eixo no qual as disciplinas fazem referência aos fundamentos da pesquisa e atuação do psicólogo, disciplinas que abordam a história da Psicologia e suas escolas, englobando as dimensões epistemológicas, teóricas e metodológicas. A formação em Psicologia expressa a atuação diversificada na prática desse profissional em seus distintos cenários, permitindo exercer atividades em programas de promoção de saúde e de qualidade de vida, conforme mencionado no quadro 1.

Embora seja possível notar algumas mudanças nos cursos analisados, já apontado a formação do psicólogo para atuação no SUS, percebemos que o profissional de Psicologia ainda é formado, principalmente, para lidar com a patologia. As repercussões desse modelo suscitam dicotomias importantes, gerando distanciamento entre a formação recebida e o que o cotidiano oferece como campo privilegiado de articulações culturais e políticas. Desse modo, as práticas passam a configurar lacunas entre os modelos de atuação e o contexto, estabelecendo-se a partir de modelos positivoindividualizantes, sobretudo através de atendimentos clínicos de cunho individualintimista. (Campos \& Guarido, 2007).

\section{CONSIDERAÇÕES FINAIS}

O SUS, ao propor um modelo de reorientação de atenção à saúde a partir da desconstrução de modelos assistenciais - os quais são centrados apenas na produção de 
procedimentos destinados à cura de doenças -, e implementar serviços de saúde atentos aos contextos de vida da população, provoca reformulações no conceito de saúde e nas práticas de saúde. Para que tais transformações tornem-se efetivas, é incontestável a necessidade de mudanças no campo da formação dos profissionais que constituem 0 SUS, inclusive a dos os psicólogos. Embora essa necessidade já tenha sido constatada e discutida por diversos pesquisadores no campo da Psicologia, percebe-se que a formação em Psicologia ainda está pouco inserida nestas discussões e, de modo geral, não possibilita a construção de práticas nesses serviços de forma reflexiva e crítica (Dimenstein, 2000; Spink, 2003; Ferreira Neto, 2004; Guareschi e cols., 2010; entre outros).

Ao estabelecermos como objetivo desta pesquisa o estudo dos cursos de graduação em Psicologia de Alagoas e sua relação com a formação para o trabalho no SUS, buscamos atentar para o caráter local dessas configurações. Os resultados encontrados a partir da análise curricular não divergiram do que vem sendo apontado sobre a formação em Psicologia no Brasil em pesquisas anteriores, citadas neste estudo. Assim como no cenário nacional, os cursos do Estado de Alagoas seguem evidenciando uma ênfase individualista, que se conjuga a concepções de sujeito, saúde e cuidado fortemente vinculadas a modelos biomédicos, conquanto possamos identificar alguns pontos de ruptura com esse modelo e abertura para as práticas da saúde coletiva, conforme proposta do SUS.

Destaca-se, nos quatro cursos estudados, a ênfase na identificação de doenças e a centralidade de abordagens psicodiagnósticas no que se refere à saúde. Ao apontarmos esse aspecto não pretendemos sugerir que tais abordagens não contribuam para a formação em Psicologia; mas entendemos que tanto sua priorização enquanto a forma como são trabalhadas devem ser sempre alvo de problematizações, assim como qualquer outro aspecto do currículo. Acreditamos que esses conteúdos precisam ser discutidos a partir de uma reflexão crítica e da análise das possibilidades de uma real contribuição às práticas psicológicas, que colocam em questão inclusive as identidades e as especificidades da Psicologia. A Psicologia não deve ser caracterizada como uma "ciência das doenças" ou das psicopatologias. Deve-se repensar o viés positivista puramente centrado no corpo para explicação dos processos de saúde e doença na formação e atuação em Psicologia.

Nos currículos dos cursos estudados também se apresentaram discussões referentes à integralidade, à consideração de diversos fatores no processo saúde-doença, à atuação em equipes interdisciplinares, à promoção de saúde e ao SUS. Ressalta-se também que estas discussões situaram-se, em sua maioria, no eixo 3 , que, por sua vez, em apenas um dos cursos equipara-se, em termos do número de disciplinas, com o eixo 1,0 que indica a necessidade de potencializar esses aspectos nos currículos. Os programas dos cursos analisados acompanham as Diretrizes Curriculares Nacionais, indicando a abordagem de cenários diversificados, a atuação multiprofissional e a capacitação profissional com base generalista; entretanto a formação ainda enfatiza uma clínica individualista, embora - campo revele a aproximação com conhecimentos acerca do SUS e com o princípio da integralidade de atenção à saúde, o que indica algumas mudanças no modelo de ensino vigente.

\section{REFERÊNCIAS}

Araújo, D., Miranda, M. C. G., \& Brasil, S. (2007). Formação de profissionais de saúde na perspectiva da integralidade. Revista Baiana de Saúde Pública, 31 (1), 20-31.

Benevides, R. (2005). A psicologia e o Sistema Único de Saúde: quais interfaces? Psicologia \& Sociedade, 17 (2), 21-25.

Brasil. (2009). O SUS de A a Z: garantindo saúde nos municípios (3 $3^{a}$ ed.). Brasília: Editora do Ministério da Saúde.

Brito-Silva, K, Bezerra, A. F. B. \& Tanaka, O. Y. (2012). Direito à saúde e integralidade: uma discussão sobre os desafios e caminhos para sua efetivação. Interface, 16 (40), 249-260.

Campos, F. C. B., \& Guarido, E. L. (2007). O Psicólogo no SUS: suas práticas e as necessidades de quem 0 procura. In: M. J. P. Spink. (Org.). A Psicologia em diálogo com o SUS: prática profissional e produção acadêmica. São Paulo: Casa do Psicólogo.

Ceccim, R. B., \& Feuerwerker, L. (2004). Mudança na graduação das profissões de saúde sob o eixo da integralidade. Cadernos de Saúde Pública, 20 (5), 14001410.

Dimenstein, M. (2000). A cultura profissional do psicólogo e o ideário individualista: implicações para a prática no 
campo da assistência pública à saúde. Estudos de Psicologia, 5 (1), 95-121.

Dimenstein, M. D. B. (1998). O psicólogo nas Unidades Básicas de Saúde: desafios para a formação e atuação profissionais. Estudos de Psicologia, 3 (1), 53-81.

Ferreira Neto, J. L. (2004). A formação do psicólogo. Clínica, social e mercado. São Paulo: Escuta.

Foucault, M. (2002). A Psicologia de 1850 a 1950. In: Ditos e Escritos I - Problematizações do Sujeito: Psicologia, Psiquiatria e Psicanálise (2 $2^{\underline{a}}$ ed., pp. 133-151). Rio de Janeiro: Forense Universitária.

Guareschi, N. M. F., Dhein, G., Reis, C., Machry, D., \& Bennemann, T. (2010). A Psicologia e a formação do profissional da saúde para o SUS: um estudo a partir dos currículos de cursos de psicologia do Rio Grande do Sul. In: N. M. F. Guareschi, A. Scisleski, C. Reis, G. Dhein, \& M. A. Azambuja. (Orgs.). Psicologia, formação, política e produção em saúde. Porto Alegre: EDIPUCRS.

Guareschi, N. M. F., Dhein, G., Reis, C., Machry, D., \& Bennemann, T. (2009). A formação em Psicologia e o profissional da Saúde para o SUS (Sistema Único de Saúde). Arquivos Brasileiros de Psicologia, 61 (3), 35-45.

Lima, M. (2005). Atuação psicológica coletiva: uma trajetória profissional em Unidades Básicas de Saúde. Psicologia em Estudo, 10 (3), 431-440.

Mattos, R. A. (2001). Os sentidos da integralidade: algumas reflexões acerca de valores que merecem ser defendidos. In: R. Pinheiro \& R. A. Mattos. (Orgs.). Os sentidos da integralidade na atenção e no cuidado à saúde (pp. 39-64). Rio de Janeiro: IMS, UERJ/ ABRASCO.

Nascimento, M. L., Manzini, J. M., \& Bocco, F. (2006). Reinventando as práticas psi. Psicologia \& Sociedade, $18(1), 15-20$.
Prado Filho, K., \& Martins, S. (2007). A subjetividade como objeto da(s) Psicologia(s). Psicologia \& Sociedade, 19 (3), 14-19.

Scarparo, H. B. K., \& Guareschi, N. M. F. (2007). Psicologia social comunitária profissional. Psicologia \& Sociedade, 19 (2), 100-108.

Spink, M. J. P. (2003). Psicologia da Saúde: a estruturação de um novo campo de saber. In: M. J. P. Spink. (Org.). Psicologia Social e Saúde: práticas, saberes e sentidos. Petrópolis: Vozes.

Spink, M. J. P., \& Matta, G. C. (2007). A prática profissional Psi na Saúde Pública: configurações históricas e desafios contemporâneos. In: M. J. Spink. (Org.). A Psicologia em diálogo com o SUS: prática profissional e produção acadêmica. São Paulo: Casa do Psicólogo.

Vasconcelos, C. M., \& Pasche, D. F. (2009). Sistema Único de Saúde. In: G. W. S. Campos, M. C. S. Minayo, M. Akerman, M. Drumond Jr., \& I. M. Carvalho. (Orgs.). Tratado de Saúde Coletiva (2ª ed). São Paulo: Hucitec.

Veiga-Neto, A. (2002). Cultura e Currículo. Revista Contrapontos, 2 (4), 43-51.

Recebido em 09/07/2012 Aceito em 19/11/2012
Endereço para correspondência:
Simone Maria Hüning. Universidade Federal de Alagoas-UFAL. Curso de PsicologiaICHCA. Tabuleiro dos Martins, CEP 57082-900, Maceió-AL, Brasil. E-mail: simonehuning@yahoo.com.br. 